University of Nebraska - Lincoln

DigitalCommons@University of Nebraska - Lincoln

Faculty Publications - Textiles, Merchandising and Fashion Design

Textiles, Merchandising and Fashion Design,

Department of

2010

Structure and properties of ultrafine silk fibers produced by Theriodopteryx ephemeraeformis

Narendra Reddy

University of Nebraska - Lincoln, nreddy3@unl.edu

Yiqi Yang

University of Nebraska - Lincoln, yyang2@unl.edu

Follow this and additional works at: https://digitalcommons.unl.edu/textiles_facpub

Part of the Art and Design Commons

Reddy, Narendra and Yang, Yiqi, "Structure and properties of ultrafine silk fibers produced by Theriodopteryx ephemeraeformis" (2010). Faculty Publications - Textiles, Merchandising and Fashion Design. 22.

https://digitalcommons.unl.edu/textiles_facpub/22

This Article is brought to you for free and open access by the Textiles, Merchandising and Fashion Design, Department of at DigitalCommons@University of Nebraska - Lincoln. It has been accepted for inclusion in Faculty Publications - Textiles, Merchandising and Fashion Design by an authorized administrator of DigitalCommons@University of Nebraska - Lincoln. 


\title{
Structure and properties of ultrafine silk fibers produced by Theriodopteryx ephemeraeformis
}

\author{
Narendra Reddy ${ }^{1}$ and Yiqi Yang ${ }^{1,2,3}$
}

1. Department of Textiles, Clothing, \& Design, 2. Department of Biological Systems Engineering, and 3. Nebraska Center for Materials and Nanoscience, University of Nebraska-Lincoln

Corresponding author - Yiqi Yang, 234 HECO Building, East Campus, University of Nebraska-Lincoln, Lincoln, NE 68583-0802, USA; email yyang2@unl.edu

\begin{abstract}
Theriodopteryx ephemeraeformis commonly known as bag worms produce ultrafine silk fibers that are remarkably different than the common domesticated (Bombyx mori) and wild (Saturniidae) silk fibers. Bag worms are considered as pests and commonly infect trees and shrubs. Although it has been known that the cocoons (bags) produced by bag worms are composed of silk, the structure and properties of the silk fibers in the bag worm cocoons have not been studied. In this research, the composition, morphology, physical structure, thermal stability, and tensile properties of silk fibers produced by bag worms were studied. Bag worm silk fibers have considerably different amino acid contents from those of the common silks. The physical structure of the bag worm silk fibers is also considerably different compared with B. mori and common wild silk fibers. Bag worm's silk fibers have lower tensile strength (3.2 g/denier) and Young's modulus (45 g/denier) but similar breaking elongation (15.3\%) compared with B. mori silk. However, the tensile strength and Young's modulus of bag worm fibers are similar to those of the common $\mathrm{Sa}$ turniidae wild silk fibers. Bag worm silk fibers could be useful for some of the applications currently using the B. mori and wild silk fibers.
\end{abstract}

\section{Introduction}

Bombyx mori (mulberry) and silk produced by the insects from the saturniidae family such as Antheraea assa- mensis (muga), Antheraea mylitta (tasar), and Phylisomia ricini (eri) are the most common types of silks in current use [1-3]. Mulberry silks have excellent properties such as good tensile strength, high elongation, and moisture regain and are extensively used for textile, medical, and biotechnology applications [1,4-7]. Although wild silks generally have inferior properties than $B$. mori silk, wild silks are reared for commercial silk production due to their unique properties and also to provide employment and income to native habitants where these cocoons are found $[8,9]$. It has also been recently reported that wild silks have better potential for tissue engineering applications than B. mori silk [10].

In addition to the common saturniidae silks, several other uncommon saturniidae insects such as Argema mittrei that produce cocoons with unique properties are being considered for commercial silk production [9]. It has also been reported that some of the uncommon $s a$ turniidae insects are easier to rear, produce larger cocoons and have properties similar or better than those of B. mori silk [11]. For instance, silk fibers produced by Coscinocera hercules moths were found to have fineness and tensile properties very similar to those of $B$. mori silk fibers [11].

Besides the saturniidae family, silks produced by insects that belong to different species have been studied for their structure and properties. Silks produced by honey bees are reported to have a coiled-coil structure consisting of four proteins with low molecular weights, 
unlike most of the common silks that have large repetitive proteins arranged in the form of a $\beta$-sheet [12]. Similarly, it was reported that Hydropsyche siltalai produces the weakest silk, but it has good elongation and can double its length before breakage [13]. Silk produced by various types of spiders has also been extensively studied for their outstanding properties and applications in various areas [14].

Bag worms named due to the bag-like cocoons they produce belong to the order Lepidoptera and family Pshyhidae that contains approximately 1,000 species [15]. Bag worms are considered as pests and generally infect ever green plants such as cedar, juniper, spruce, and pine. Although the production of "silken threads" from bag worms has been reported, the structure and properties of the silk produced by bag worms are not known. The cocoons produced by bag worms provide elevated temperature than the outside atmosphere to the insect that accelerates development. The inside of the bag worm cocoon has a temperature of $13^{\circ} \mathrm{C}$ compared with the outside temperature of $8{ }^{\circ} \mathrm{C}$ [16]. The bag worm cocoons also protect the insects from natural enemies [15].

In this research, we have studied the structure and properties of the bag worm silk fibers in comparison to the properties of $B$. mori and common saturniidae silks. Data available from previous studies have been used for B. mori and the saturniidae silks to include properties reported by various authors.

\section{Materials and methods}

Bag worm (Theriodopteryx ephemeraeformis) cocoons used for this study were found on Juniper plants in Lincoln, Nebraska during the summer of 2009. Chemicals used for degumming were purchased from VWR International, Bristol, CT.

\section{Degumming}

The bagworm cocoons were treated in chloroform to remove any waxes. Treated cocoons were then degummed in water containing $0.5 \%(\mathrm{w} / \mathrm{w})$ sodium carbonate and $10 \%$ ethylene diamine solution at $85^{\circ} \mathrm{C}$ for $1 \mathrm{~h}$ with a solution to cocoon ratio of 20:1. The degummed cocoons were thoroughly washed in warm water and dried under ambient conditions.

\section{Morphology}

Images of the cocoons were obtained using a digital camera. Size of the cocoons was determined using normal rulers. The undegummed cocoons and fibers obtained after degumming were observed using a scanning electron microscope (SEM) (Hitachi S 3000N variable pressure SEM) to determine the longitudi- nal and cross-sectional features of the cocoons and degummed fibers. Samples were sputter coated with gold palladium and observed in the SEM at a voltage of $20 \mathrm{kV}$. Twenty fibers from three different SEM pictures were measured to determine the average and \pm one standard deviation of the fiber diameter.

\section{Composition}

Degummed bag worm silk fibers were analyzed for the proportion of various types of amino acids using a Hitachi L-8800A amino acid analyzer. Fibers were dissolved in $6 \mathrm{~N}$ hydrochloric acid under argon atmosphere for $20 \mathrm{~h}$ at $110^{\circ} \mathrm{C}$. The samples were then evaporated to dry and then redissolved in $200 \mu \mathrm{L}$ of $0.02 \mathrm{~N} \mathrm{HCl}$. Fifty microliters of the solution was injected automatically into the amino acid analyzer to determine the type and proportion of amino acid. Corrections were made to the amount of internal standard (Norleucine) to minimize dilutional errors.

\section{Thermal behavior}

Bagworm silk fibers were observed in a Thermogravimeric Analyzer (TGA) (Sigma Model 701) to understand the thermal behavior of the fibers in comparison to $B$. mori silk fibers. Samples were heated at $10^{\circ} \mathrm{C} / \mathrm{min}$ up to $600^{\circ} \mathrm{C}$ in the TGA.

\section{$X$-ray diffraction}

X-ray diffraction studies were conducted to understand the physical structure of the bag worm silk fibers in terms of \% crystallinity and positions of the diffraction peaks. Fibers were grounded in a Wiley mill to pass through a $20 \mu \mathrm{m}$ mesh. Powdered fiber samples were used for X-ray analysis to eliminate the influence of any preferred orientation of the crystals on the X-ray diffraction patterns. Using powdered samples is a common technique used for analyzing X-ray diffraction of fibers. The powdered fibers were compressed to form a pellet.

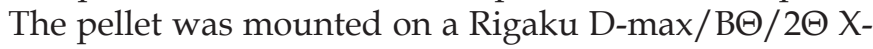
Ray diffractometer (Rigaku Americas, Woodlands, TX) with Bragg-Brentano parafocusing geometry, a diffracted beam monochromator, and a copper target $\mathrm{X}$-ray tube set to $40 \mathrm{kV}$ and $30 \mathrm{~mA}$. Diffraction intensities were determined for $2 \theta$ values ranging from $5^{\circ}$ to $40^{\circ}$. The $\%$ crystallinity of the fibers was determined by integrating the area under the crystalline peaks after subtracting the background and air scatter using the program MICROCAL ORIGIN.

\section{Tensile properties}

Fibers were conditioned under standard conditions $\left(21^{\circ} \mathrm{C}\right.$ and $65 \%$ relative humidity) for at least $24 \mathrm{~h}$ before 


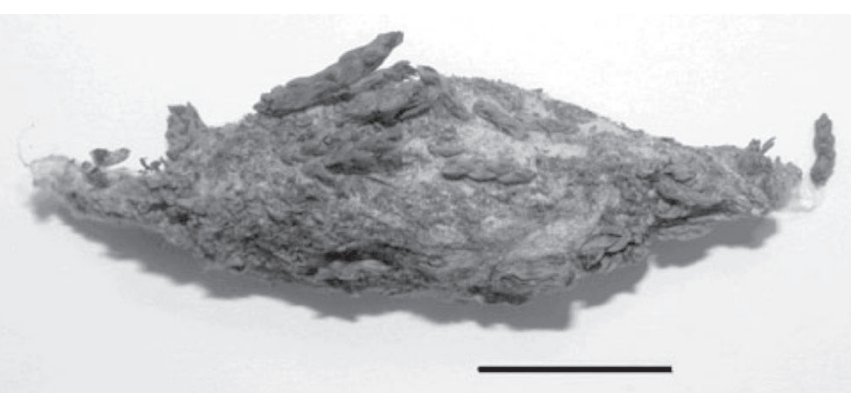

Figure 1. Digital image of an intact, undegummed bag worm cocoon shows plant materials attached to the cocoon. Scale bar is $1 \mathrm{~cm}$.

tensile testing. Fineness of the fibers in terms of denier (weight in grams per 9,000 meters) was determined by precisely weighing a known length of fibers determined using a standard ruler. Fiber samples were tested for their tensile properties using an Instron tensile tester (Model 4444). A gauge length of 1 inch and crosshead speed of $18 \mathrm{~mm} / \mathrm{min}$ were used for testing. About $30 \mathrm{fi}-$ bers were tested, and the average and standard deviations are reported.

\section{Results and discussion}

\section{Bag worm cocoons}

Bag worm cocoons are oval shaped, about $3.5 \mathrm{~cm}$ in length, dark brown in color and covered with plant material on the outside as seen from Figure 1. In addition to providing protection from the outside environment, the plant materials on the cocoon also act as a camouflage to predators. Empty cocoons without the plant materials on the surface had an average weight of $85 \mathrm{mg}$, much lower than the weight of common silk worms. The inside of the cocoon was similar to a woven fabric with some loose white silk fibers (indicated by arrow) as seen from Figure 2. The silk fibers in the cocoon were compact, and it was very difficult to remove fibers from the cocoon before degumming. SEM image of the cocoon in Figure 3 shows that the cocoon consists of long and fine fibers. There is no preferred orientation, and the fibers run above and below each other creating a network structure that provides good strength to the cocoons.

\section{Morphology of the bag worm silk fibers}

Bag worm silk fibers obtained after degumming had smooth and clean surface as seen from Figure 4 . The fibers in Figure 4 had an average diameter of $2.9 \pm 1 \mu \mathrm{m}$. Figure 5 shows that the fibers have a solid cross section, and most of the fibers are circular unlike B. mori silk fibers that have a triangular cross section.

\section{Composition of bag worm silk fibers}

Bag worms have a very unique composition of amino acids than B. mori and the common wild silks as seen from Table 1. Alanine and glycine are the two major amino acids in B. mori $(74 \%)$ and wild silks $(62-73 \%)$, but these two amino acids account for only $6.6 \%$ in bag worm silks $[2,3]$. The bag worm silks have much higher content of lysine, leucine, phenylalanine, threonine, cysteine, and histidine than B. mori and the common wild silks. The amino acids glycine, alanine, serine, and threonine are reported to be in the crystalline region whereas the other amino acids are reported to be in the amorphous region for $B$. mori silk $[2,3,17,18]$. The ratio of glycine/alanine is reported to determine the crystallographic form of the proteins. The glycine/alanine ratio of bag worm silk fibers is 0.27 , but 1.52 for $B$. mori silk fibers indicating that the bag worm silk should have a considerably different crystallographic form [19, 20]. The ratio of amino acids in the crystalline and noncrystalline region is referred to as the disorder ratio and is related to the tensile properties and hydrophilicity of the fibers [18]. Disorder ratio of bag worm silks is 0.18, much lower than the ratio for B. mori silk (6.7) suggesting that bag worm silk will have inferior tensile properties compared with $B$. mori silk.

Three groups of silks have been recognized based on the amino acid composition. The first group consists of silks in which the alanine, glycine, and serine content is $60 \%$ or higher [21]. This class includes B. mori and the other wild silk fibers in Table 1 . The second group of silks contains a combination of alanine, glycine, and serine in addition to either proline or glutamine accounting for $60 \%$ of the amino acids [21]. The third group consists of silks in which no two amino acids account for $60 \%$ or higher amino acid content. Bag worm silk belongs to the third group of insects [21]. The type and amount of amino acids in insects are said to be determined by the diet, cost of producing the silk, and the ecology of the insects [21]. The unique amino acid composition of the bag worms could be due to the lack of proper diet, season in which the cocoons were collected or the inherent biology of the insects.

Table 2 provides a comparison of the various amino acid ratios in the bag worm silks with $B$. mori and the common wild silks. Bag worm silk had relatively more uniform distribution of acidic and basic groups compared with $B$. mori silk which has much higher amounts of acidic amino acids than basic groups. However, the bag worm silk contains substantially higher number of hydrophilic groups than hydrophobic groups compared to the other silks in Table 2. Bag worm silks have much lower ratio of glycine/alanine than the other silks in Table 2 . Tables 1 and 2 show that bag worms produce silk containing unique ratio of amino acids than $B$. mori and common wild silks. 


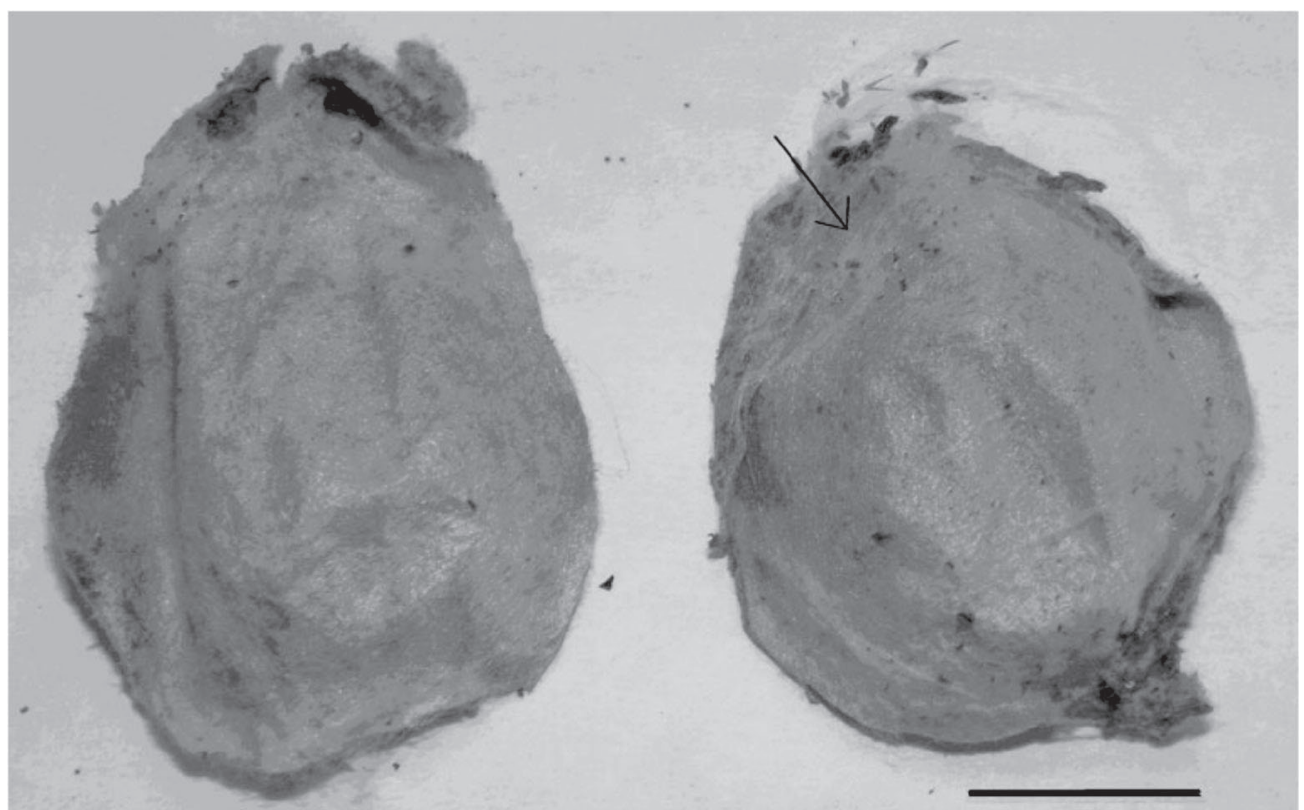

Figure 2. Image of the inside surface of the bag worm cocoon has a fabric-like appearance. The inside surface had a layer of loosely attached white silk fibers (arrow). Scale bar is $1 \mathrm{~cm}$.

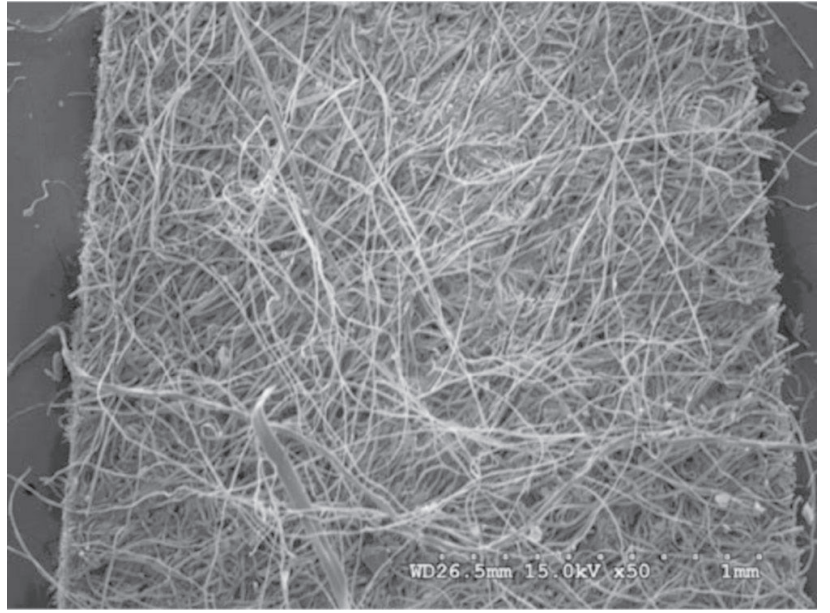

Figure 3. SEM image of the surface of the bag worm cocoon shows the random orientation of the silk fibers that helps to create a firm cocoon.

\section{Thermal decomposition}

The thermal decomposition of the bag worm silk fibers is compared with that of B. mori silk in Figure 6 . Both the bag worm and B. mori silk have similar thermal behavior up to about $200{ }^{\circ} \mathrm{C}$ and have a weight loss of about $12 \%$. The bag worm silk shows a slightly higher weight loss between 200 and $300{ }^{\circ} \mathrm{C}$. However, the weight loss of the bag worm silk is much lower than that of B. mori silk above 300 and up to about $600{ }^{\circ} \mathrm{C}$. At $600{ }^{\circ} \mathrm{C}$, the weight loss of the bagworm silk was about $82 \%$ compared with $91 \%$ for the B. mori silk. The better thermal stability of the bag worm silk to decomposition at high temperatures should mainly be due to the differ-

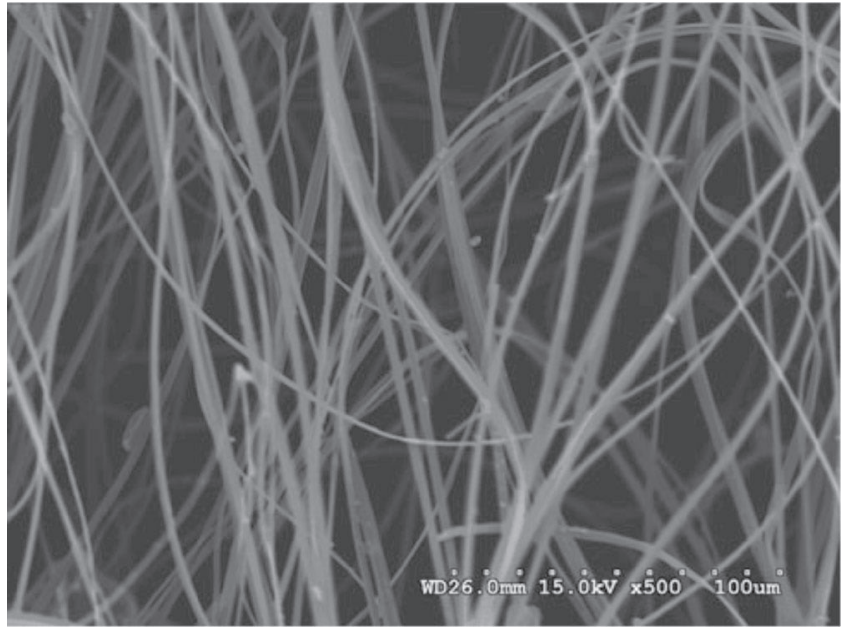

Figure 4. Degummed bag worm silk fibers have a clean and smooth surface with an average diameter of $2.9 \mu \mathrm{m}$.

ent amino acid compositions of the bag worm silks compared with B. mori silk despite the bag worm silks having lower \% crystallinity Figure 7.

\section{Physical structure}

X-ray diffractogram of the bag worm silk fibers is compared with B. mori silk fibers in Figure 7. The bag worm silk fibers show similar diffraction peak compared with the B. mori silks. Both silks show a single prominent diffraction peak at $2 \theta$ angle of $19.8^{\circ}$ corresponding to the 201 plane. The 201 plane in B. mori is reported to correspond to a d-spacing of $4.43 \AA$. Five types of fibroin have been classified based on the spacing and intensities 
Table I. Comparison of the amino acid composition of the bag worm silk fibers with B. mori and three varieties of common wild silks

\begin{tabular}{llllll}
\hline Amino acids & \multicolumn{5}{l}{ \% Amino acids } \\
\cline { 2 - 6 } & Bag worm & B. mori & A. mylitta & A. pernyi & P. ricini \\
\hline Lysine & II.I & 0.2 & 0.2 & 0.2 & 0.2 \\
Alanine & 5.2 & 29.4 & 32.1 & 34.1 & 36.3 \\
Glycine & 1.4 & 44.6 & 40.6 & 27.7 & 29.4 \\
Serine & 3.6 & 12.1 & 4.2 & 9.9 & 8.9 \\
Tyrosine & 6.8 & 5.2 & 4.6 & 6.8 & 5.8 \\
Leucine & 10.9 & 0.7 & 0.8 & 0.7 & 0.7 \\
Aspartic acid & 7.8 & 1.3 & 0.9 & 6.1 & 3.9 \\
Arginine & 5.5 & 0.5 & 2.4 & 5.0 & 4.1 \\
Glutamic acid & 14.5 & 1.0 & 1.3 & 1.3 & 1.3 \\
Phenylalanine & 7.2 & 0.1 & 0.3 & 0.3 & 0.2 \\
Threonine & 5.1 & 0.9 & 0.3 & 0.2 & 0.2 \\
Valine & 4.8 & 2.4 & 1.7 & 1.5 & 1.3 \\
Cysteine & 4.7 & 0.1 & 0.2 & 0.1 & 0.1 \\
Histidine & 3.7 & 0.1 & 0.8 & 0.7 & 0.8 \\
Proline & 3.9 & 0.8 & 2.2 & 2.2 & 2.0 \\
Isoleucine & 2.2 & 0.8 & 0.6 & 0.5 & 0.5 \\
Methioine & 1.3 & 0.2 & 0.3 & 0.3 & 0.3 \\
\hline
\end{tabular}

Data for B. mori and the wild silks are from [2, 3]

Table 2. Comparison of the amino acid ratios in bag worm silk fibers with the common silks

\begin{tabular}{lllll}
\hline Ratio & Bag worm & B. mori & A. mylitta & P. ricini \\
\hline Basic/acidic & 0.91 & 0.65 & 0.97 & 1.30 \\
Hydrophilic/hydrophobic & 1.9 & 0.28 & 0.44 & 0.35 \\
Glycine/alanine & 0.3 & 1.58 & 0.81 & 0.80 \\
\hline
\end{tabular}

Data for B. mori and wild silks are from reference [2, 3]

of diffraction arcs [22]. Silk produced by the Psychidae family has been classified under a different group than B. mori and Saturniidae silks but with similar fiber repeat distance [22]. More than $80 \%$ of the polypeptides in $B$. mori fibroin have been reported to consist of Gly-X (GX) sequence in the crystalline domain whereas the sequence of amino acids in bag worm silks is not known [23, 24].

The $\%$ crystallinity of bag worm silk fibers was found to be $33.8 \%$ from the diffractogram shown in Figure 7 compared with $30-40 \%$ for B. mori and common wild silks $[25,26]$. It is reported that the crystalline regions in B. mori and common wild silks consists of the amino acids glycine, alanine, serine, and threonine [23-27]. However, the sum of these amino acids in bag worm silks is only $15.2 \%$ whereas the \% crystallinity was found to be $33.8 \%$. This suggests that the crystalline regions in bag worm silks consist of other amino acids than those found in B. mori and common wild silks. The considerably different amino acid compositions of bag worm silks as given in Table 1 and the previous report that classified fibroin from Psychidae family in a different group than $B$. mori, and wild silks based on the differences in diffraction patterns substantiates our assumption.

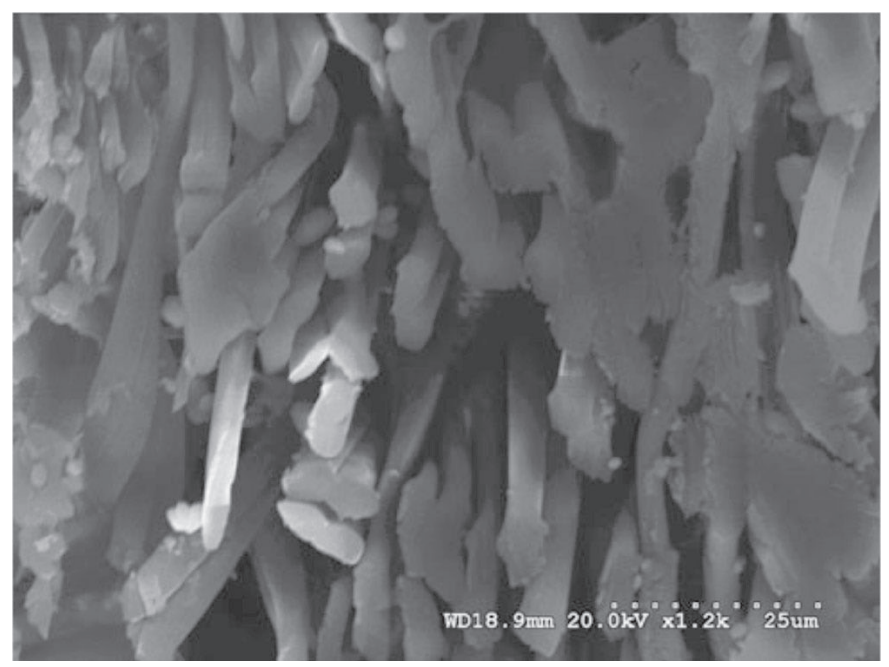

Figure 5. Bag worm silk fibers have a solid cross section, and most of the fibers are circular.

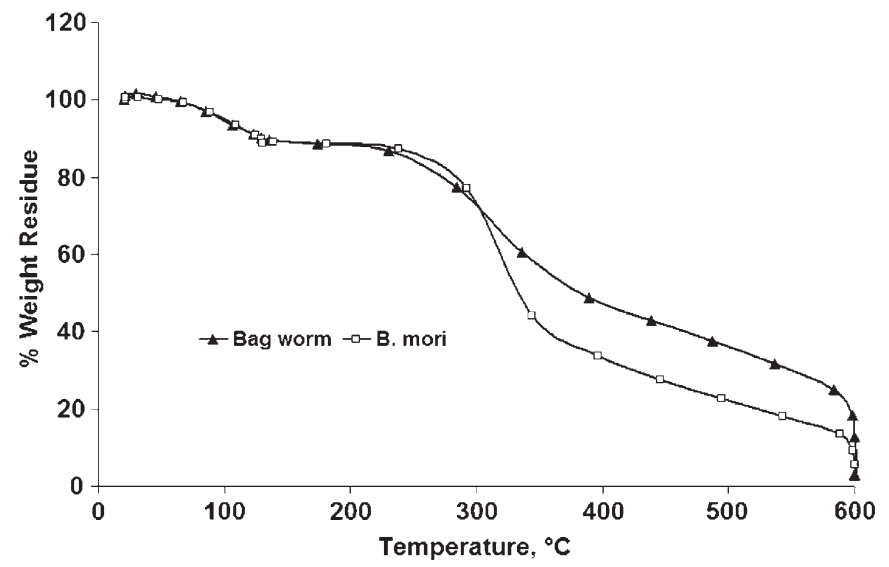

Figure 6. Thermal decomposition of bag worm silk fibers compared with $B$. mori silk fibers.

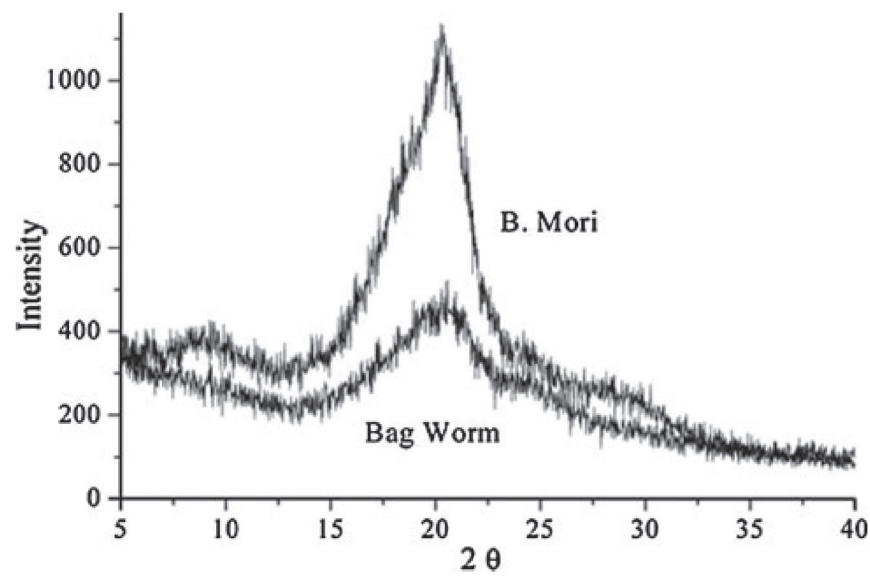

Figure 7. Diffractogram of bag worm silk fibers compared with B. mori silk fibers. 
Table 3. Tensile properties of bag worm silk fibers compared with B. mori, A. mylitta, and P. ricini silks

\begin{tabular}{lllll}
\hline Fiber & Bag worm & B. mori & A. mylitta & P. ricini \\
\hline Fineness, denier & $0.9 \pm 0.1$ & $0.4-1.1$ & $4.7-10.7$ & $2.3-3.6$ \\
Breaking tenacity, g/denier & $3.2 \pm 1.0$ & $4.3-5.2$ & $2.5-4.5$ & $1.9-3.5$ \\
Breaking elongation, \% & $15.3 . \pm 6.2$ & $10.0-23.4$ & $26-39$ & $24-27$ \\
Young's modulus, g/denier & $45 \pm 12$ & $84-121$ & $66-70$ & $29-31$ \\
\hline
\end{tabular}

Data for B. mori and wild silks are from references $[2,3]$

\section{Tensile properties}

Bag worm silk fibers have fineness similar to those of $B$. mori silk, but are much finer than the wild silks as seen from Table 3. However, the breaking tenacity of the bag worm fibers at $3.2 \mathrm{~g} /$ denier is lower than that of $B$. mori and in the range of breaking tenacity for the wild silk fibers. Breaking elongation of the bag worm silk fibers is similar to that of B. mori silk but considerably lower than that of the wild silks. The modulus of bag worm fibers is also considerably lower than the modulus of $B$. mori and A. mylitta but similar to the modulus of $P$. ricini silk as seen from Table 3. Lower breaking tenacity of the bag worm fibers is most likely due to the low amounts of crystalline amino acids as indicated by the low disorder ratio. Size of the insects, bag worms being considerably smaller than that of B. mori or the wild silk caterpillars, probably makes it difficult for the bag worms to secrete the crystalline amino acids. Low modulus of the bag worm fibers indicates that the fibers are soft to touch. Although the breaking tenacity of the bag worm fibers is lower than that of $B$. mori silk, natural cellulose fibers such as cotton have similar (2-3 g/denier) breaking tenacity but much lower (8-9\%) breaking elongation. This indicates that bag worm fibers will be suitable for commercial applications.

\section{Conclusions}

Bag worms produce very firm oval-shaped cocoons with average weight of about $85 \mathrm{mg}$. Silk fibers in the cocoons had an average diameter of about $2.9 \mu \mathrm{m}$. The fibers are composed of very distinct amino acids than those in the common silk worms. The alanine and glycine content in bag worm silk fibers was $6.6 \%$ compared with $74 \%$ for $B$. mori silk suggesting that bag worm silks have a considerably different crystallographic forms than those of $B$. mori silk. Bag worm silk fibers also show better stability to thermal degradation than those of B. mori silk from 300 to $600^{\circ} \mathrm{C}$. Tensile strength of bag worm silk fibers is considerably lower, but elongation and modulus are similar to that of B. mori silk. This article shows that bag worm silk has unique composition and properties than that of the common silk fibers. Other species in the Pshyhidae family may have also unique structure and properties.

Acknowledgments - The authors acknowledge the financial support from the Agricultural Research Division at the University of Nebraska-Lincoln, USDA Hatch Act, and MultiState Project S1026 for completing this research.

\section{References}

1. Robson RM (1998) In: Lewin M, Pearce EM (eds) Handbook of fiber chemistry. Marcel Dekker Inc, New York

2. Sen K, Babu MK (2004) J Appl Polym Sci 92:1080

3. Sen K, Babu MK (2004) J Appl Polym Sci 92:1098

4. Kameda T, Tsukada M (2006) Macromol Mater Eng 291:877

5. Acharya C C, Ghosh SK, Kundu SC (2009) Acta Biomater 5(1):429

6. Kearns V, MacIntosh AC, Crawford A, Hatton PV (2008) Top Tissue Eng 4:1

7. Takeshita H, Ishida K, Kamiishi Y, Yoshii F, Kume T (2008) Macromol Mater Eng 283:126

8. Kakati LN, Chutia BC (2009) Trop Ecol 50(1):137

9. Razafimanantosoa $\mathrm{T}$, Ravoahangimalala OR, Craig CL (2006) Madag Conserv Dev 1(1):34

10. Acharya C, Ghosh SK, Kundu SC (2008) J Mater Sci Mater Med 19:2827

11. Reddy N, Yang Y (2010) Int J Biol Macromol 46(4):419

12. Sutherland TD, Weisman S, Trueman HE, Sriskantha A, Trueman JWH, Haritos VS (2007) Mol Biol Evol 24(11):2424

13. Brown SA, Ruxton GD, Humphries A (2004) J N Am Benthol Soc 23(4):771

14. Saravanan D (2006) J Text Appar Technol Manag 5(1):1

15. Rhainds M, Davis DR, Price PW (2009) Annu Rev Entomol 54:209

16. Weiss HB (1914) Psyche 21(1):45

17. Bhat NV, Nadiger GS (1980) J Appl Polym Sci 25:921

18. Perez-Rigueiro J, Elices M, LLoraca J, Viney C (2001) J Appl Polym Sci 82:53

19. Rajkhowa R, Gupta VB, Kothari VK (2000) J Appl Polym Sci 77:2418

20. Shaw JTB, Smith SG (1961) Biochem Biophys Acta 52:305

21. Craig CL, Hsu H, Kaplan D, Pierce NE (1999) Int J Biol Macromol 24:109

22. Warwicker JO (1960) J Mol Biol 2:350

23. Zhou C, Confalonieri F, Jacquet M, Perasso R, Li Z, Janin J (2001) Proteins Struct Funct Bioinf 44:119

24. Gage LP, Manning RF (1980) J Biol Chem 255(19):9444

25. Lotz B, Cesari C (1979) Biochemie 61:205

26. Zhao C, Yao J, Masuda H, Kishore R, Asakura T (2003) Biopolymers 69:253

27. Dobb MG, Frazer RDB, Macrae TP (1967) J Cell Biol 32:289 